\title{
Propuesta didáctica para la enseñanza de Geografía en quinto grado de primaria con el uso de Google Earth
}

Joaquín Alonso Flores Sánchez

lg.alonsofloress@gmail.com

Universidad Autónoma del Estado de Hidalgo

Bonifacio Doroteo Pérez Alcántara

bonipa62@gmail.com

Universidad Autónoma del Estado de México

Recibido:24 de marzo del 2020

Aceptado: 24 de abril del 2020

\section{Resumen}

El trabajo presenta el diseño de una propuesta didáctica realizada y aplicada con alumnos de quinto grado, en una escuela primaria rural del estado de Veracruz, con el uso de Google Earth como alternativa de enseñanza y aprendizaje de algunos temas geográficos, lo anterior ante el requerimiento de los Planes y Programas de Estudios 2011 sobre la implementación de estrategias que incorporen el uso de las Tecnologías de la Información y Comunicación (TIC) para apoyar diferentes asignaturas. El artículo, busca abonar a dichos requerimientos y tiene como prioridad que los estudiantes hagan uso del poco equipo de que dispone su escuela y manejen información geográfica, a través de este programa, mediante el uso de las TIC, de manera interactiva y atractiva, de tal forma que se geste un aprendizaje significativo, reflejado en el rendimiento académico de los alumnos. El procedimiento consistió en un diagnóstico breve con el docente y alumnos del grupo, para conocer la percepción que tienen, y la importancia que otorgan al uso de esta herramienta como alternativa de enseñanza y aprendizaje. Con los resultados obtenidos, se formuló y corrió la propuesta, en el marco del constructivismo en que operan los programas, con apoyo del modelo ASSURE, evidenciando mayor interés por el conocimiento geográfico y su rendimiento escolar.

Palabras claves: Educación primaria, Google Earth, Geografía. 


\title{
Didactic proposal for the teaching of Geography in fifth grade of primary school with the use of Google Earth
}

\begin{abstract}
The document presents the design of a didactic proposal applied in students of fifth grade in a rural primary school, located in the state of Veracruz, with the use of Google Earth as an alternative for teaching and learning geographical topics, the above due to the requirements of the 2011 study plans and programs in the implementation of strategies that incorporates the use of Information and Communication Technologies (ICT) to support different subjects. The article seeks to contribute to these requirements and has as a priority that students make use of the little equipment available at the school and learn geographical information through this program with the use of ICT in an interactive and attractive way, so that they have a significant learning that will be reflected in their academic performance. The procedure consists of a brief diagnosis with the teacher and students of the group, to know their perception, and the importance that the use of this tool gives as an alternative of teaching and learning. With the results obtained the proposal was formulated within the framework of the constructivism, in which the programs operate, with the support of the ASSURE model, evidencing a greater interest towards the geographical knowledge and therefore in school performance
\end{abstract}

Keywords: Primary education, Google Earth, Geography

\section{Introducción}

La propuesta, deriva de las observaciones realizadas durante la práctica docente de uno de los autores, realizada en el ciclo escolar 2017-2018, en la escuela primaria rural Benito Juárez García, de la localidad de Tigrillos, municipio de Apazapan, Veracruz, donde se pudo constatar la trasmisión de contenidos geográficos, por parte del docente a sus estudiantes, de manera memorística y descriptiva con un mapa impreso. A raíz de lo anterior, se formularon al docente una serie de preguntas al finalizar sus clases y se pudo constatar que, para él, es la forma correcta de brindar instrucción a sus alumnos. Sin embargo, de ese modo se logra una asimilación parcial del conocimiento por parte de los alumnos en un ambiente de aprendizaje muy poco favorable. 
En la actualidad, derivado de las nuevas políticas y programas educativos, diversos autores, (Llanos, 2014; Rodríguez, 2006), señalan que, la educación geográfica, debe responder a la complejidad de un espacio geográfico en permanente transformación. Debido a que los estudiantes están inmersos en la sociedad de la información, donde puede acceder a un gran volumen de información, que se les debe enseñar a seleccionar y aplicar, de forma correcta, en su vida cotidiana.

En este contexto, el docente de educación primaria debe actualizarse y hacer uso de estrategias que apoyen la enseñanza de contenidos de las asignaturas, por el trabajo que desempeña, debe conocer y emplear los 10 saberes digitales básicos:

1. Saber usar dispositivos (computadoras, tabletas, impresoras, proyectores y equipo de sonido), 2. Administrar archivos, 3. Usar programas y sistemas de información especializados, 4. Crear y manipular contenido de texto y texto enriquecido, 5. Crear y manipular conjuntos de datos, 6. Crear y manipular medios y multimedia, 7. Comunicarse en entornos digitales, 8. Socializar y colaborar en entornos digitales, 9. Ejercer y respetar una ciudadanía digital y 10. Literacidad digital (Ramírez \& Casillas, 2017, págs. 130-131).

Lo anterior se vincula con la inclusión de habilidades digitales, las cuales, de acuerdo con la Secretaría de Educación Pública (SEP, 2016), deben ser implementadas en el aula con los recursos tecnológicos disponibles, para enseñar los contenidos de diferentes asignaturas. Por tales razones, los docentes mexicanos adquieren el reto de dominar las competencias necesarias para el uso eficiente de las TIC y su puesta en práctica en el aula.

Así surgió la idea de diseñar una propuesta didáctica, para la enseñanza de Geografía, con el uso de Google Earth y aplicarla con los estudiantes del quinto grado, matriculados en la escuela de referencia, buscando clases más dinámicas e interactivas, mediante el uso de los medios electrónicos a su alcance y con ello, favorecer la motivación, el aprendizaje y por qué no, su rendimiento académico. Lo anterior con apoyo del modelo de diseño instruccional ASSURE, cuyos detalles se brindan en el apartado metodológico. 
La propuesta es congruente con los Planes y Programas de Estudios realizados por la SEP, en el año 2011, los cuales se abordan desde el constructivismo, que, como lo indican (Duffy y Cunningham, 1996), es el proceso de construcción de conocimientos e interpretaciones, donde el estudiante asimila la información para otorgarle un nuevo significado, mediante la confrontación, el intercambio y negociación permanente del saber.

Ante tales argumentos, surge la siguiente interrogante, ¿Cómo perciben los alumnos y el docente de quinto año de primaria el uso y sus beneficios de las TIC y el programa Google Earth, para potencializar su aprovechamiento y elevar el rendimiento académico en la asignatura de Geografía?

\section{Metodología}

El enfoque del trabajo es de tipo cualitativo, entendido como el "conjunto de prácticas interpretativas que hacen al mundo, visible y a su vez lo transforman y lo convierten en una serie de representaciones observables" (Grinnell y Unrau, 2005, pág. 10). Específicamente se trata de un trabajo de corte descriptivo, sustentado en el registro e interpretación de la naturaleza y composición de un fenómeno o proceso (Tamayo, 2002), en el momento en que ocurren las cosas. Lo anterior, al amparo del modelo ASSURE, el cual, a pesar de tener sus raíces teóricas en el conductismo, tiene rasgos constructivistas, al preocuparse por la participación comprometida del estudiante (Benites, 2010).

Lo anteriormente señalado, permitió orientar la investigación como intervención educativa, centrada en el conjunto de acciones planteadas más adelante (tabla 1), para un determinado contexto social y educativo (Gómez y Gallón, 2005); donde se busca que el estudiante, con ayuda del docente, logre un desarrollo integral.

Acto seguido, por acuerdo con las autoridades y el docente, se decidió trabajar con el $5^{\circ}$ grado de primaria, donde se apreciaron mayores limitantes en el proceso de enseñanza con temas de Geografía, cuando colaboramos con el docente, como parte del proceso de experimentación al realizar los estudios de la Especialidad en Docencia que imparte la Universidad Autónoma del Estado de Hidalgo. 
Se pensó en el modelo instruccional ASSURE (figura1) para llevar a cabo los procesos educativos de forma sistemática, en la enseñanza de la Geografía y diseñar actividades atractivas para los alumnos, además que fuesen medibles y evaluables los resultados obtenidos

Figura 1. Modelo instruccional A.S.S.U.R.E. y sus etapas para la implementación

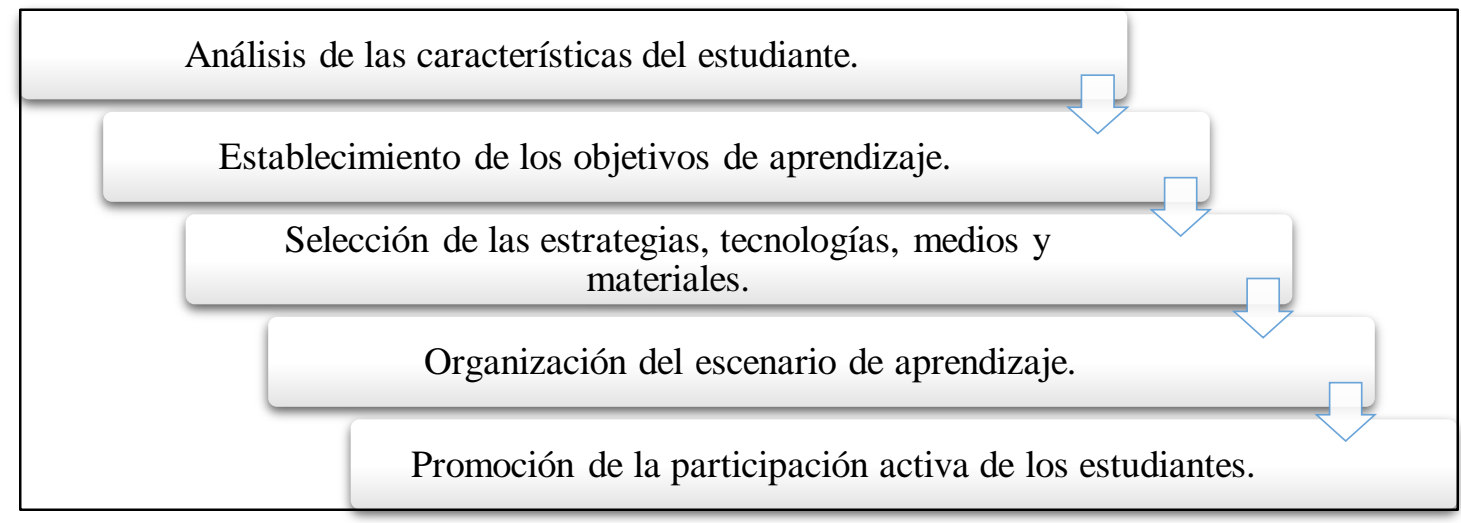

Fuente: (Aziz, 1999).

La recogida de datos fue a través de una encuesta, con la finalidad de reconocer el acercamiento que poseen los estudiantes con las TIC y Google Earth, disposición para su uso y posibles dificultades, a manera de diagnóstico. Se encuesto al docente para conocer su opinión valorar la pertinencia de la propuesta que se pretendía realizar. Es importante mencionar que dicho instrumento, se basa en los aportes realizados por EDUTEKA (2019).

Se trabaja con los objetivos de la materia de Geografía, específicamente para dar cumplimiento a los temas de la Unidad 4 ¿Cómo localizo? haciendo uso de Google Earth como herramienta tecnológica de apoyo. Con todo ello se diseñó la estrategia didáctica, como se observa en la figura 2, considerando, las competencias que deben desarrollar el docente y sus estudiantes, así como los recursos didácticos de los que puede hacer uso el maestro para llevar a cabo la sesión de clase, y las acciones que competen a ambos en el aula. El escenario de aprendizaje es el salón de clase y de computación, sin dejar de lado, la parte de evaluación, expresada en una lista de cotejo (tabla 2). 
Figura 2. Planeación, supervisión, valoración de los procedimientos de la Estrategia Didáctica

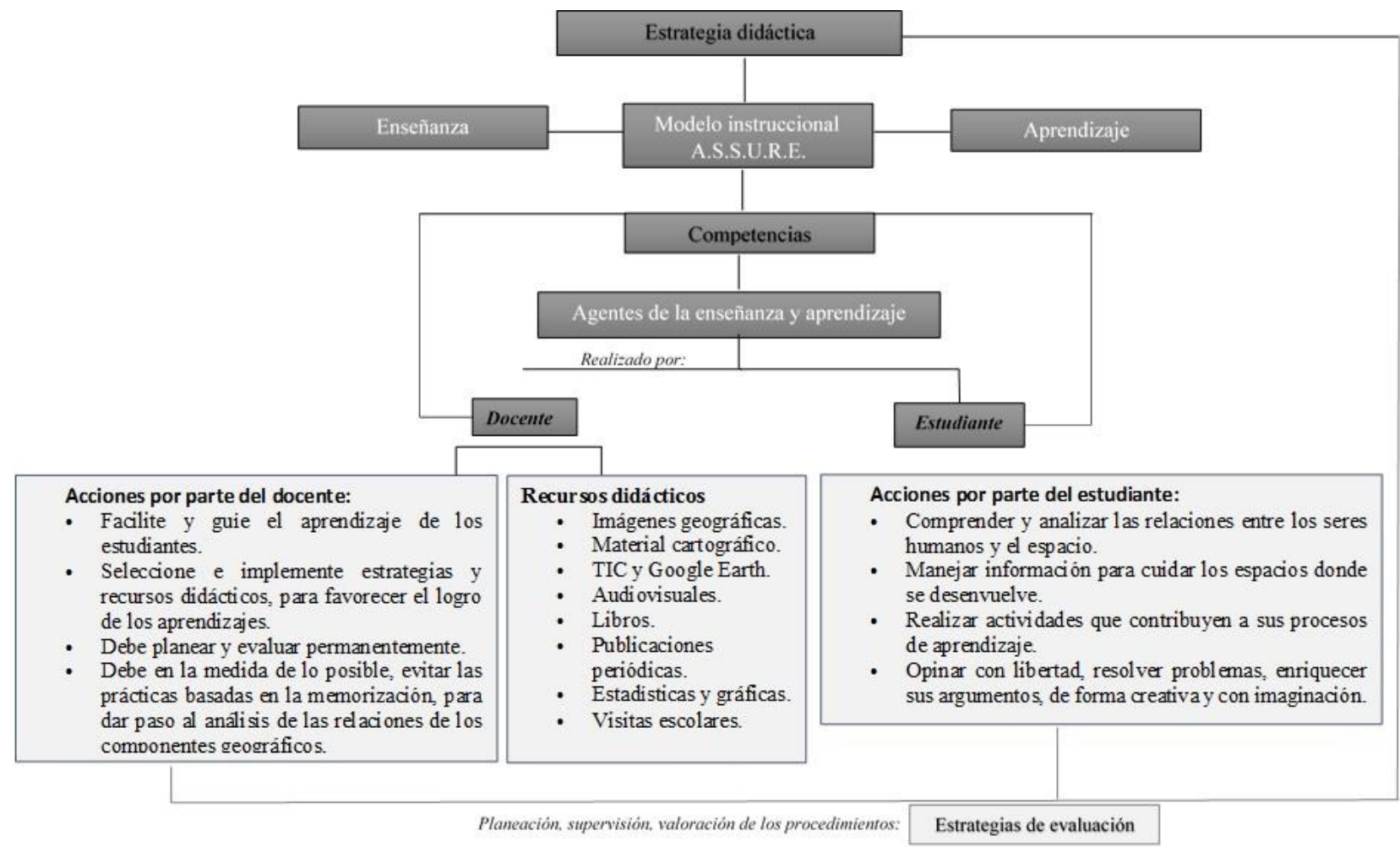

Fuente: Elaborado con base a Feo, 2010; SEP, 2011.

\section{Referentes teóricos}

Si bien es cierto que el modelo ASSURE tiene referentes de corte conductista, la investigación está sustentada en el enfoque constructivista, el cual busca promover en los estudiantes la reflexión de sus experiencias para construir su propia percepción del mundo en el que viven. Además, porque justamente en el constructivismo se sustentan los actuales planes y programas de estudio del nivel básico en México, a tal grado que, en este país "las escuelas están empezando a ser un slogan o imagen de marca [...] pues desde finales del siglo pasado, podemos observar que casi todas las teorías educativas y/o instruccionales parecen haber abierto sucursales constructivistas" (Pozo, 2005, págs. 61-62).

Es así como, a los niños y las niñas, se les enseña -que por sí mismos o en ocasiones acompañados del docente-, a desarrollarse en el ámbito personal y social, por medio del lenguaje y comunicación (SEP, 2011), pero también a indagar las formas de construir su propio conocimiento. 
Las TIC constituyen el insumo complementario para esta actividad, y "giran en torno a tres medios básicos: la informática, la microelectrónica y las telecomunicaciones; pero no lo hacen de forma aislada, sino de manera interactiva e interconexionadas, lo que permite conseguir nuevas realidades comunicativas" Cabero (1998, pág. 198). Eso pretende la estrategia, en congruencia con la SEP (2011) cuando sugiere los medios y entornos digitales para comunicar ideas e información e interactuar con otros y resolver problemas educativos.

De este modo, con el uso de las TIC en educación, se comienzan a generar cambios significativos, los cuales resultan positivos en el rendimiento académico de los niños y las niñas que cursan cualquier grado de Educación Básica Martínez (2010). Sin embargo, se debe tener en cuenta que las estrategias de aprendizaje no siempre buscan que el estudiante aborde directamente los conceptos del curso, sino que sólo reconozca lo que ya sabe, o simplemente necesite repasar los contenidos establecidos en los Planes y Programas de Estudios Garza (2012).

Google Earth es una herramienta poderosa para el cambio, actualización y mejoramiento de los procesos educativos, los docentes deben valoren las conexiones entre dicha herramienta y los diferentes aspectos de su trabajo profesional: nuevas teorías de aprendizaje, estrategias de aula actualizadas, cumplimiento de lineamientos o estándares curriculares y métodos de evaluación (EDUTEKA, 2008).

Dicha herramienta se convierte en alternativa viable para el desarrollo de ciertas actividades propuestas en el libro de texto de Geografía, específicamente en el bloque uno, en el que se encuentra la lección cuatro denominada ¿Cómo localizo? donde se propone el uso de Google Earth, pues se espera que, por este medio, los estudiantes del quinto grado tengan mayor interés, en el tema, le otorguen un mejor significado y obtengan un óptimo desarrollo en el aprendizaje y se vea reflejado en su rendimiento académico, entendido como "el cumplimiento de las metas, logros y objetivos establecidos en el programa o asignatura que cursa un estudiante, expresado a través de calificaciones, que son resultado de una evaluación que implica la superación o no de determinadas pruebas, materias o cursos" (Caballero y Palacio, 2007, pág. 98).

El rendimiento académico, no significa una mejor calificación, este incorpora factores internos como; inteligencia, personalidad, actitudes, estilos de aprendizaje y familiares, están relacionados de forma directa con el estudiante (Antolín, 2013; Planck, 2014). Además, se suman 
otros de carácter externo, y estos tienen su origen en el contexto e historia de vida de cada uno de los estudiantes, la comunidad educativa, la personalidad de los docentes y su desempeño en el aula frente a los estudiantes, el entorno escolar y el nivel socioeconómico (Garbanzo, 2007; Garijo, 2016), todos estos muy imprtantes en el área de estudio, donde predominan las actividades agropecuarias, pocos servicios (agua, luz, drenaje, internet limitado, una clínica rural, transporte público) y la oferta educativa es de preescolar a secundaria (modalidad de telesecundaria), la población total en 2010 era de menos de 700 personas (INEGI, 2011) y a la fecha sigue por debajo de mil habitantes, debido a la migración interna y hacia los Estados Unidos de Norteamérica, que no cesa.

\section{Resultados}

Recordemos que el diagnóstico, como primera fase del modelo para la propuesta, se llevó a cabo valorando la percepción de los alumnos, sobre el uso de las TIC y el manejo que tienen de herramientas informáticas y sobre los beneficios que creen les puede otorgar el uso de dichas herramientas en sus procesos escolares. La figura 3 muestra la disposición de los niños para conocer más acerca de dichos recursos, específicamente para el abordaje de los temas de Geografía. Destaca que el 67\% de los encuestados tienen interés y están de acuerdo en conocer, aprender y manejar las TIC y los programas informáticos, pues a pesar de su corta edad, saben que con estos apoyos, pueden seleccionar y aplicar información para complementar su formación académica.

En contraparte, el 33\% restante, consideran que las clases se imparten mejor sin el uso de las tecnologías, debido a que temen por su seguridad, según sus argumentos. al verse vulnerables por estar expuestos a que cualquier otra persona los pueda ver y de esa forma se coloquen en una situación de riesgo, mostrando un desconocimiento absoluto de los recursos y su verdadera utilidad académica, o la falta de información por parte de los padres y maestros, asociado a la edad propia de los niños, quienes oscilan entre los 11 y 12 años. 
Figura 3. Disposición por Conocer, Usar las TIC y los Programas Informáticos por parte de los alumnos.

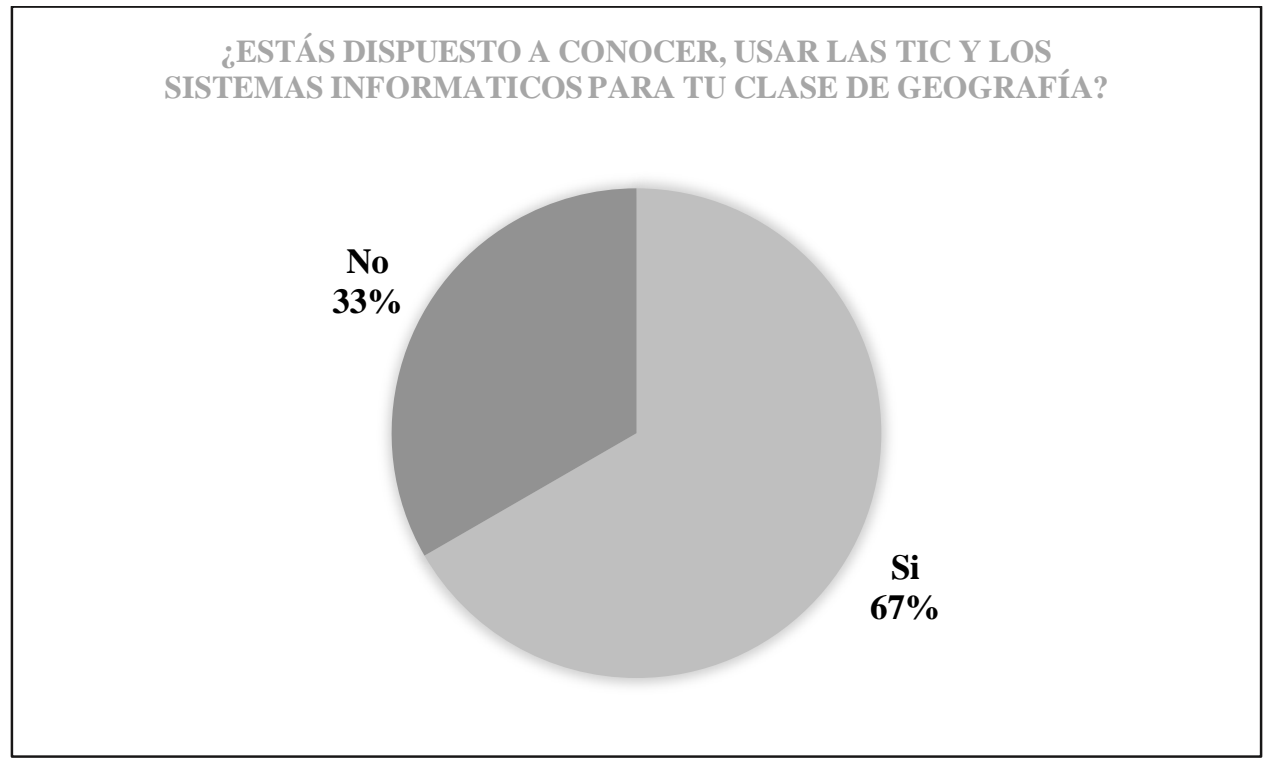

Fuente: Elaboración propia

Respecto a los beneficios identificados por los alumnos con la implementación de las TIC y los programas informáticos, reconocen la importancia de emplearlos en su salón de clase, con la posibilidad de aprender de manera diferente los diversos contenidos de los libros. El 83\% de los niños y las niñas, refieren la pertinencia de su posible uso y ocupación, como parte de sus procesos didácticos, sin embargo, $17 \%$ considera que implementar las tecnologías y programas informáticos en su salón de clase, no generará ningún cambio como se abordan los temas (figura 4). A pesar de eso, todos coincidieron que por lo menos se pruebe su implementación en una de las asignaturas escolares, para ver cómo funcionan otras formas de enseñar y aprender. Y así se generó la propuesta.

Figura 4. Beneficios del uso de las TIC y los Programas Informáticos. Fuente: Elaboración propia

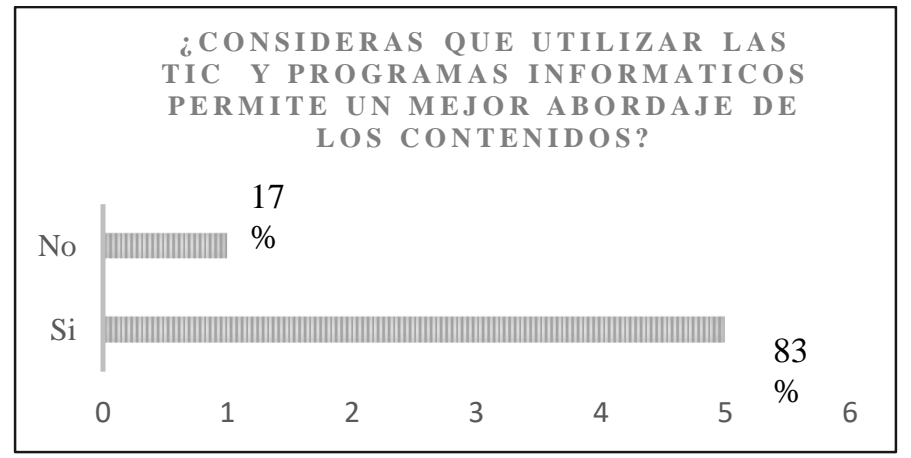

Revista RedCA junio-septiembre 2020 ISSN: 2594-2824 Vol. 2 Núm. 7 
En lo que concierne a la encuesta realizada al docente que atiende a los estudiantes del quinto grado, sobre el uso, manejo, conocimiento de las TIC y los programas informáticos para abordar los contenidos de Geografía, reconoce lo siguiente:

- No cuenta con recursos didácticos suficientes y estrategias atractivas y en ocasiones resulta difícil abordar determinados temas de geografía o cualquier otra asignatura.

- Posee conocimiento limitado y hace uso de las TIC, con fines educativos, con paquetería de Microsoft Office: reproductores de audio, video y softwares como edilim y cuadernia.

- El maestro asegura estar dispuesto a actualizarse en el uso y manejo de las TIC, para mejorar su práctica educativa, en beneficio del aprendizaje de sus alumnos.

- La dificultad a las que enfrenta es el desconocimiento de programas informáticos que le permitan hacer de su enseñanza algo innovador para ser aplicado con los estudiantes.

Lo referente a la implementación en el aula del programa informático Google Earth, el docente menciona lo siguiente:

- No ha empleado Google Earth para la enseñanza de la Geografía, sin embargo, ha escuchado hablar de él y sabe qué se usa para localizar lugares de cualquier parte del planeta.

- Al referir a los beneficios del uso del Google Earth, menciona que, está interesado en aprender a usarlo y apropiarse, pues le resulta interesante aprender cosas nuevas y es consciente de que la sociedad actual lo demanda.

- El docente considera que sus alumnos, podrán acceder a información para descargar, visualizar y consultar fotos, imágenes satelitales, relieves en 3D, algo que los libros de texto ofrecen solo de manera impresa.

- Concibe que, usar Google Earth en el aula, puede modificar la manera en la que actualmente enseña y de esa forma, se interesarían más en aprender.

- Lo que concierne a las dificultades y los requerimientos para el uso del Google Earth, el docente menciona que sólo se trata de saber utilizar la computadora, y que esta sea de fácil acceso, pues en ocasiones debido a la falta de mantenimiento, es lenta.

- En lo relacionado a la disposición, para él, aprender cosas nuevas es parte de lo que lo hace crecer como docente, aunque las limitaciones locales siempre influyen. 


\section{Secuencia didáctica}

La estructura para la secuencia didáctica, se basó en los planteamientos de Feo (2010) y las fases del modelo instruccional A.S.S.U.R.E., con los cuales se construye la tabla 1, donde se ilustra la propuesta para abordar la lección cuatro del bloque uno, del libro de texto de Geografía, de quinto grado, de tal manera que el estudiante empiece con algunas aplicaciones y pueda generar soluciones de acuerdo con la teoría constructivista según los requerimientos del constructivismo en que se sustenta su educación. El uso de Google Earth, favorece el manejo de la información geográfica y fortalece su comprensión, en este caso, al implementar acciones que permiten aplicar el primer principio de la ciencia: La localización.

Tabla 1 Secuencia Didáctica, para abordar los contenidos de la lección 4, ¿Cómo localizo?, bloque uno, de Geografía.

Incorporación del Google Earth para elevar el rendimiento académico, bajo la técnica de estudios de casos.

Nombre de la Escuela: Benito

Juárez García.

Nombre del docente:

Grupo: $5^{\circ}$ A.

Nombre de la estrategia: Uso del Google Earth para localizar lugares nacionales, estatales y locales de interés, mediante la técnica de estudios de casos, para promover el rendimiento académico de los estudiantes del quinto grado.

Tema: ¿Cómo localizo?
Contexto: La escuela atiende durante el ciclo escolar 2019-2020, una matrícula de 54 estudiantes, de los cuales seis integran el grupo de quinto grado.

Objetivo: Que el estudiante aprenda a manejar el Programa Informático Google Earth como herramienta y pueda localizar distintos lugares como: países, ciudades $\mathrm{y}$ localidades a partir de las coordenadas

\section{Duración total: 1 hora, 30} minutos.
Sustentación teórica: A partir del constructivismo, mediante contenidos significativos y trascendentes, los cuales están vinculados con su contexto, su experiencia previa y condiciones de vida, para la oportuna apropiación de una serie de referentes que consoliden un pensamiento crítico y reflexivo. Revista RedCA junio-septiembre 2020 ISSN: 2594-2824 Vol. 2 Núm. 7 
geográficas y la división política del continente americano, empleando la técnica de estudios de casos, para la mejora en su rendimiento académico.
Desde la Ciencia que nos ocupa, se retoma el primer principio del método geográfico, el cual alude a la localización y distribución, en congruencia con el tema 4 del Programa de Geografía de quinto grado de primaria.

\section{Contenidos de la secuencia didáctica:}

1. Latitud y longitud; 2. Altitud; 3. División política continental, nacional, estatal y local; 4. Apliquemos lo que aprendí; 5. Evaluación.

\section{Actividades:}

Se explica a los estudiantes, el objetivo de la lección que se aborda.

Se hace uso del recurso visual (proyector), con el que cuenta su salón de clase, para explicar la definición de latitud, longitud y altitud.

Se lleva a cabo un dibujo de la forma geoide del planeta, para reforzar la explicación anterior.
Recursos y medios:

\section{Multisensorial: docente.}

Multisensorial: docente y estudiante; visual: planisferio.

Multisensorial: docente; visual: pizarrón.
Estrategia de

evaluación:

Momento:

Actividad:

Introducción al tema.

Técnica:

Observación.

Instrumento:

Guía de

observación.

\section{Tiempo estimado: 25 minutos.}

Se les solicita a los estudiantes activar la pestaña "Ver" y dar clic en la opción "cuadrícula" para poder visibilizar las coordenadas geográficas.
Multisensorial: docente; tecnológico: pc y Google Earth.
Actividad:

Visualización de coordenadas.

\section{Técnica:} Observación y estudio de caso.
Lista de cotejo.

Se le proporciona a cada estudiante la actividad a desarrollar.

Actividad 1: Con ayuda del Google Earth cada estudiante identifica los lugares localizados en Veracruz: municipales, regionales y locales, que se encuentran en la
Multisensorial: $\quad$ Actividad: docente y estudiante; Localización de impreso: hojas de papel con las indicaciones precisas.

Multisensorial: estudiante; tecnológico:

pc y Google Earth, e impreso: hojas de papel. sitios del estado de Veracruz.

Técnica: Estudio de caso.

Instrumento:

Registro de notas.
Desarrollo

Revista RedCA junio-septiembre 2020 ISSN: 2594-2824 Vol. 2 Núm. 7 
hoja proporcionada por el

docente.

Tiempo estimado: 40 minutos.

$\mathrm{Se}$ les menciona a los estudiantes la actividad a desarrollar y se les proporciona el material.

Multisensorial: docente; impreso: hoja de papel; tecnológico:

pc y Google Earth.

Actividad 2: Con ayuda de Google Earth, cada estudiante identifica cinco lugares de su interés -internacionales, nacionales, municipales, regionales y locales- los cuales deberán anotar.

Actividad 3: Se dará respuesta -en plenaria- a las siguientes preguntas:

1. ¿De qué manera se representa en un globo terráqueo la latitud? 2. ¿De qué manera se representa en el globo terráqueo la longitud? 3. ¿En función de que, se mide la altitud de un lugar determinado?

\section{Tiempo estimado: 25 minutos.}

Efectos esperados: los estudiantes mediante la técnica de estudios de caso y el uso del Google Earth, serán capaces de manejar información geográfica, relacionada con el principio de localización, inherente a la ciencia geográfica, desde el contexto internacional hasta el local, con la intención de que sean capaces de reconocer la importancia de saber ubicarse con ayuda de las coordenadas geográficas, mediante la herramienta citada.

Observaciones: Si se requiere, se realizan las observaciones pertinentes sobre la aplicación de la secuencia, con la intención de retroalimentar al grupo y reforzar los conocimientos.

Fuente: Elaborada con base a Feo, 2010; SEP, 2011 y el modelo A.S.S.U.R.E.

\section{Estrategias de evaluación}

Todo proceso educativo exige llevar a cabo un ejercicio de evaluación, para conocer el nivel de logro de los objetivos o propósitos planeados desde un inicio. Además, la evaluación permite recabar información útil para valorar los procesos de enseñanza y aprendizaje de manera 
formativa, sumativa y en su caso correctiva, identificando las áreas de oportunidad con los niños y eventualmente, poder atenderlas.

Feo (2010), diseña estrategias que permiten evaluar el acto educativo donde el docente emplea los instrumentos elaborados por él mismo, creados en función de lo que éste espera enseñar y lo que se espera que aprendan los estudiantes, que participan en la clase. Al momento de elaborar un instrumento de carácter evaluativo, por parte del docente, se deben considerar las siguientes normas:

- Ser útil para facilitar información respecto a las virtudes y defectos que se presentan durante la puesta en marcha de un plan de clase.

- Proponer soluciones de mejora.

- Ser clara y precisa desde el momento en el que se redacta.

- Poseer compromisos explícitos, como la presentación de los resultados de manera trasparente, pues se puede generar un plan de mejora (Mora, 2004)

La tabla 2 contiene el instrumento de evaluación de la propuesta didáctica, la cual cuenta con una escala numérica de 10, 8 y 6 , que permite valorar el nivel de logro de las actividades programadas y puestas en marcha por el docente frente al grupo de quinto grado, para abordar el tema de estudio, retomando algunos aspectos sugeridos por Mora (2004) y Feo (2010) 


\section{Tabla 2 Lista de Cotejo para la Evaluación de las Actividades Propuestas en la Secuencia Didáctica}

\section{Actividades programadas \\ Escala numérica de evaluación \\ Actitudes por parte del docente para el abordaje \\ 10 \\ 8 \\ 6 del tema}

1. Explico con claridad los objetivos del tema.

2. Tengo la capacidad de visualizar el alcance de los objetivos.

3. Selecciono los contenidos que imparto siguiendo ciertos criterios (relevancia, utilidad, interés de los estudiantes, etc.).

4. Calculo el tiempo que voy a dedicar a cada apartado de la secuencia didáctica.

5. Estimo el tiempo que los estudiantes requieren para el aprendizaje de los contenidos.

6. Pongo en marcha los métodos docentes que empleo para el abordaje de la lección.

7. Tengo claridad en lo que deseo que los estudiantes aprendan al abordar la lección.

8. Preparo las actividades que el estudiante debe realizar durante la sesión de clase.

9. Tengo en cuenta los recursos de los que puedo disponer para impartir la lección.

10. Decido los criterios y procedimientos de evaluación de aprendizaje en función de las características de la lección.

11. Fomento en los estudiantes una autoevaluación para conocer lo que han aprendido.

12. Incorporo los estilos de aprendizaje de mis estudiantes al momento de realizar la secuencia didáctica.

13. Verifico que los recursos que voy a utilizar en clase estén disponibles.

14. Elaboro un guion de lo que voy a tratar en clase.

15. Preparo ejercicios, preguntas y/o problemas para que los estudiantes trabajen en clase.

16. Preparo ejemplos y/o aplicaciones para aclarar el contenido de clase.

17. Pienso en la forma de evaluar lo aprendido por los estudiantes, durante la sesión de clase. 
Fuente: Elaborado con base a Feo, 2010 y Mora, 2004.

\section{Conclusiones}

Derivado de la experiencia vivida en la comunidad de Tigrillos, municipio de Apazapan en el estado de Veracruz, es posible asegurar que en el medio rural y muchas veces en el medio urbano, la enseñanza de la Geografía sigue siendo de carácter memorístico, al abrigo del modelo tradicional, comúnmente alimentado por la comodidad o la falta de preparación y conocimiento geográfico del personal docente que trabaja frente agrupo.

Mediante el diagnóstico realizado, se pudo confirmar que, en su mayoría, los alumnos y el maestro, tienen interés por explorar y conocer el uso de las TIC y programas informáticos como Google Earth, al considerarlos benéficos y atractivos para abordar los temas de Geografía y aunque las limitantes son muchas, con estrategias como esta, se promueve el uso de los equipos y otros insumos con los que cuenta la escuela para generar nuevas experiencias de aprendizaje.

Ante dichas circunstancias, es imperioso la programación y preparación de actividades de enseñanza y aprendizaje con el uso de dichas herramientas, que promuevan mayor interés por parte de los alumnos y consecuentemente un mejor aprovechamiento académico.

Las limitaciones tecnológicas son evidentes en la escuela, sin embargo, derivado del ejercicio anterior, el docente se encuentra en la mejor disposición para diseñar e implementar en sus clases, las secuencias didácticas que sean requeridas, no solo en la materia de Geografía, sino en cualquier otra, por las bondades que ofrece, tanto para la planeación y organización, como para promover los aprendizajes de formas alternas a las ocupadas hasta el momento. 
Después de diseñar y aplicar la secuencia propuesta con los estudiantes y el docente, queda claro que es muy útil el uso de Google Earth, para entender y comprender, no solo los ejercicios aquí planteados, es funcional para visualizar también las formas del relieve en 3D, realizar vuelos guiados sobre la superficie terrestre y comprender con mayor detalle el principio geográfico de localización.

Del mismo modo, esta herramienta puede ser útil para el manejo y comprensión de otros principios en la asignatura de Geografía, como la causalidad y la correlación, sin embargo, se requiere, para tal efecto, el apoyo de profesionales de la ciencia para la habilitación del personal docente en los distintos niveles donde se imparte la asignatura.

Un aspecto importante, en todo proceso educativo, es la forma en la que el docente y los estudiantes muchas veces llegan a acuerdos para evaluar los aprendizajes esperados respecto a los obtenidos, de allí la importancia del diseño de un instrumento claro para todos los actores involucrados, que permite valorar la puesta en marcha del plan de clase, los aciertos y errores, para de esa forma, proponer soluciones de mejora. Finalmente, la propuesta didáctica sugerida, es solo un ejemplo que ilustra las cualidades que posee Google Earth al ser empleado con fines educativos. Ahora es tarea del docente decidir en qué momento lo retoma y la forma en cómo lo aplica.

\section{Referencias bibliográficas}

Antolín, R. (2013). Motivación y Rendimiento Escolar en educación primaria. Recuperado septiembre 26, 2019, de Universidad de Almería: http://repositorio.ual.es/bitstream/handle/10835/3060/Trabajo.pdf?sequence=

Aziz, H. (1999). Assure learning through the use of the assure model. (V. C. College, Ed.) Office of Information Technology (OIT). Recuperado septiembre 10, 2019, de Research articles,

Valence.: http://www.valenciacc.edu/oit/articles/articles_detail.cfm?ID=45 
Benites, M. G. (2010). El modelo de diseño instruccional ASSURE aplicado a la educación a distancia. Tlatemoani, 1-13.

Caballero, C. C., Abello, R., \& Palacio, J. (2007). Relación de burnout y rendimiento académico con la satisfacción frente a los estudios en estudiantes universitarios. Avances en Psicología Latinoamericana, 25(2), 98-111. Recuperado octubre 11, 2019, de http://www.scielo.org.co/pdf/apl/v25n2/v25n2a7.pdf

Cabero, J. (1998). Impacto de las nuevas tecnologías de la información y la comunicación en las organizaciones educativas. En M. e. Lorenzo, Enfoques en la organización y dirección de instituciones educativas formales y no formales. Granada: Grupo Editorial Universitario. Recuperado agosto 02, 2019, de http://ardilladigital.com/DOCUMENTOS/TECNOLOGIA\%20EDUCATIVA/TICs/ T2\%20NNTT\%20Y\%20N\%20ED/CABERO\%20organizacion\%20ed..pdf

Duffy , T., y Cunningham, D. (1996). Constructivism: Implications for the design and delivery of instruction. In J. Spector, M. Merrill, M. J. Bishop, M. Bishop, \& J. M. Spector (Ed.), Handbook of Research on Educational Communications and Technology. New York: Springer. Recuperado septiembre 11, 2019, de http://homepages.gac.edu/ mkoomen/edu241/constructivism.pdf

EDUTEKA. (2012, julio 23). Google Earth en clase de Geografía. Recuperado abril 14, 2019, de EDUTEKA: http://eduteka.icesi.edu.co/articulos/GoogleEarth

Feo, R. (2010). Orientaciones básicas para el diseño de estrategias didácticas. Tendencias Pedagógicas(16), 221-236. Recuperado octubre 15, 2019, de https://revistas.uam.es/tendenciaspedagogicas/issue/download/TP16/122

Garbanzo, G. (2007). Factores asociados al rendimiento académico en estudiantes universitarios, una reflexión desde la calidad de la educación superior pública. (43- 
63, Ed.) Revista Educación, 31(1). Recuperado septiembre 14, 2019, de https://www.redalyc.org/pdf/440/44031103.pdf

Garijo, R. (2016). Analisis de los factores determinantes del bajo rendimiento academico en educación secundaria obligatoria en la Provincia de Albacente. Recuperado septiembre 17, 2019, de E-Spacio UNED: http://espacio.uned.es/fez/eserv/tesisuned:EducacionRgarijo/GARIJO_RODENAS_Ramon_Tesis.pdf

Garza, B. (2012). Modelo didáctico para el diseño de objetos de aprendizaje. Recuperado septiembre 13, 2019, de Consejo Mexicano de Investigación Educativa (COMIE): http://www.comie.org.mx/congreso/memoriaelectronica/v10/pdf/area_tematica_07/ ponencias/1462-F.pdf

Gómez, M. Á., Alzate, M. V., Arbeláez, M. C., Romero, F., y Gallón, H. (2005, juliodiciembre). Intervención y mediación pedagógica: los usos del texto escolar. Revista Colombiana de Educación(49), 83-102. Recuperado septiembre 12, 2019, de https://www.redalyc.org/pdf/4136/413635243005.pdf

Grinnell Jr. y Unrau, Y. (2005). Investigación y evaluación del trabajo social: enfoques cuantitativos y cualitativos. Nueva York, Estados Unidos: Aprendizaje Cengage. Recuperado septiembre 30, 2019, de https://scholarworks.wmich.edu/books/306

INEGI. (2011). Censo de Población y Vivienda 2010. Aguas Calientes: INEGI.

Llanos, E. (2014, enero-junio). Reflexiones acerca de la enseñanza de la geografía en el Departamento del Atlántico. Zona Proxima(20), 153-164. Recuperado abril 18, 2019, de Zona Próxima: https://www.redalyc.org/articulo.oa?id=85331022013

Martínez, R., \& Heredia, Y. (2010). Tecnología educativa en el salón de clase: Estudio retrospectivo de su impacto en el desempeño académico de estudiantes 40 universitarios del área de Informática. Revista Mexicana de Investigación Educativa 
(REMIE). Recuperado abril 20, 2019, de Revista Mexicana de Investigación Educativa.: https://nanopdf.com/downloadFile/tecnologia-educativa-en-el-salon-declase-estudio-retrospectivo-de_pdf

Mora, A. I. (2004, julio-diciembre). La Evaluación Educativa: Concepto, Periodos y Modelos. Revista Electrónica "Actualidades Investigativas en Educación", 4(2). Recuperado octubre 16, 2019, de https://www.redalyc.org/pdf/447/44740211.pdf

Planck, U. (2014). Factores determinantes del rendimiento académico de los estudiantes de la Universidad de Atacama. Estudios pedagógicos, 40(1), 25-39. Recuperado septiembre 14, 2019, de https://scielo.conicyt.cl/pdf/estped/v40n1/art02.pdf

Pozo, J. I. (2005). Aprendices y Maestros. La nueva cultura del aprendizaje (sexta ed.). Madrid, España: Alianza Editorial.

Ramírez, A., y Casillas, M. (2017). Saberes digitales de los docentes de educación básica. Una propuesta para la discusión desde Veracruz. (Primera ed.). Xalapa, Veracruz, México: Secretaría de Educación de Veracruz. Recuperado el 20 de agosto de 2019, de https://www.uv.mx/personal/albramirez/files/2015/06/Saberes-Digitales-SEVlibro-final.pdf

Rodríguez, E., Cely, A., Moreno, N., Durán, A., \& Von Prahl, A. (2006, julio-diciembre). Problemas de aprendizaje de la Geografía en alumnos de educación básica. Geoenseñanza, 11(2), 241-248. Recuperado abril 18, 2019, de GEOENSEÑANZA: https://www.redalyc.org/pdf/360/36012425010.pdf

SEP. (2011a). Guía para el maestro. Primaria. Quinto grado. In SEP, Programa de estudio 2011. Ciudad de México. Recuperado abril 30, 2019, de http://edu.jalisco.gob.mx/cepse/sites/edu.jalisco.gob.mx.cepse/files/sep_2011_progr amas_de_estudio_2011.guia_para_el_maestro_quinto_grado.pdf 
SEP. (2011b). Plan de Estudios 2011. Educación Básica. Ciudad de México. Recuperado mayo 07, 2019, de Secrearía de Educación Pública.: https://www.gob.mx/cms/uploads/attachment/file/20177/Plan_de_Estudios_2011_f. pdf

SEP. (2016). Programa de Inclusión Digital 2016 - 2017 (Primera ed.). Ciudad de México. $\begin{array}{lllll}\text { Recuperado } & \text { abril } & 18, & \text { de }\end{array}$ http://www.gob.mx/cms/uploads/attachment/file/171123/PROGRAMA_APREND E.pdf

Tamayo, M. (2002). El proceso de la investigación científica. México: Limusa-Noriega Editores. 